\title{
Magnetic Tactile Sensor for Braille Reading
}

\author{
Ahmed Alfadhel, Member, IEEE, Mohammed Asadullah Khan, Member, IEEE, Susana Cardoso, \\ Member, IEEE, and Jürgen Kosel, Senior Member, IEEE
}

\begin{abstract}
We report a biomimetic magnetic tactile sensor for Braille characters reading. The sensor consists of magnetic nanocomposite artificial cilia implemented on magnetic micro sensors. The nanocomposite is produced from the highly elastic polydimethylsiloxane and iron nanowires that exhibit a permanent magnetic behavior. This design enables remote operation and does not require an additional magnetic field to magnetize the nanowires. The highly elastic nanocomposite is easy to pattern, corrosion resistant and thermally stable. The tactile sensors can detect vertical and shear forces, which allows recognizing small changes in surface texture, as in the case of Braille dots. The 6 dots of a braille cell are read from top to bottom with a tactile sensor array consisting of 4 elements and 1 mm long nanocomposite cilia.
\end{abstract}

Index Terms-Artificial Skin, Braille, Cilia, Magnetic, Nanocomposite, Nanowires, Tactile sensor

\section{INTRODUCTION}

$\mathrm{E}$ VOLUTIONS in robotics or prosthetics are demanding an increased perception of the environment, which led to the development of tactile sensors for artificial skin application. A very interesting application for artificial skins is reading Braille characters for robots, blind people with prosthetics or to those who lost their sense of touch from a disease like neuropathy. Braille (named in 1824 after Frenchman Louis Braille) is a tactile writing system used by people who are blind or visually impaired. Noticeable progress in the field of artificial skins lead to the development of different technologies that can mimic the complex sense of touch in humans [1-4]. These artificial skins can help people with prosthetic limbs regain their sense of touch for better interaction with the surrounding environment. These skins are

Manuscript received Mar XX, 2016; revised April XX, 2016; accepted XX. Date of publication XX, 2016; date of current version XX, 2016. The associate editor coordinating the review of this paper and approving it for publication was Prof. XX.

A. Alfadhel, M. A. Khan, and J. Kosel are with the Computer, Electrical and Mathematical Sciences and Engineering Division (CEMSE), King Abdullah University of Science and Technology (KAUST), Thuwal, Saudi Arabia (E-mail: ahmed.fadhel@kaust.edu.sa; mohammedasadullah.khan@kaust.edu.sa; jurgen.kosel@kaust.edu.sa).

S. Cardoso is with the INESC-Microsystems and Nanotechnologies (INESC-MN), Rua Alves Redol, 9, 1000-029 Lisbon, Portugal and with the Physics Department, Instituto Superior Técnico, Universidade de

Lisboa, Lisbon, Portugal (e-mail: scardoso@inesc-mn.pt).

Color versions of one or more of the figures in this paper are available online at http://ieeexplore.ieee.org.

Digital Object Identifier XXXXXXXX also important for applications like health monitoring systems and smart surgical tools. Tactile sensors are the essential components of the artificial skin, and they attracted considerable attention, resulting in the development of different sensing mechanisms [4-6].

Artificial cilia sensors are attractive for researchers who try to mimic the extremely sensitive microscale cilia receptors [79]. The high aspect ratio and surface area to volume ratio of cilia allow intimate interaction with the environment.

Advances in material integration and nanofabrication technologies have enabled new directions of research and development for micro-sensor systems. Examples are nanocomposite materials, which combine the advantages of polymers like flexibility, elasticity, chemical resistance or biocompatibility with the unique properties of additives [911]. Magnetic nanocomposites have been introduced before using superparamagnetic particles [12,13]. Unlike these particles that require a high magnetic field for magnetization, which increases the power consumption and limits the possibility of integration and miniaturization, iron nanowires (NWs) have a high magnetization at remanence, due to the strong shape anisotropy, and they can be easily fabricated with a cost effective process.

In this work, we developed a tactile sensor array for Braille letter reading, which utilizes permanent magnetic and highly elastic nanocomposite artificial cilia realized on magnetic micro sensing elements. The nanocomposite is made of polydimethylsiloxane (PDMS) and iron NWs. The magnetic stray field of the cilia depends on the cilia's deflection and is detected by a giant magneto-resistive (GMR) sensor. The tactile sensor array is made of four sensing elements, which are separated by $0.8 \mathrm{~mm}$ and operate simultaneously. Each sensor has an active area of $1.25 \mathrm{~mm} \times 1.25 \mathrm{~mm}$, and consists of nine cilia that are $200 \mu \mathrm{m}$ in diameter $1 \mathrm{~mm}$ long. In general, it is easy to obtain different cilia diameters and aspect ratios with the facile fabrication process reported in this paper, enabling simple control of the sensors' performance [14]. The unique concept combining cilia and magnetic sensing allows detecting shear and vertical forces in dry or wet environments, making it suitable for applications in prosthetics, medical tools or underwater robots. The DC operated sensor can detect static and dynamic forces as well as vibration and fluid flow.

This paper is a progressive work of a previously reported tactile sensor [14,15] and a flow sensor [9] that uses a similar concept but with a high frequency magnetic sensor to detect the stray field of the cilia. Here we move to DC operation by using GMR sensors to simplify the required circuitry and to allow the realization of arrays with small sensing pixels. 


\section{METHODS}

\section{A. Sensor Concept}

The operating principle of the sensor is based on detecting the change of the magnetic stray field, created by the permanent magnetic cilia, when deflected by an external force (Fig. 1). A GMR sensor, which is simple to fabricate and can be realized on flexible substrates, is utilized to measure the change in the magnetic field [16-20]. At resting position, the stray field of the cilia affects the GMR sensor with an average magnetic field value that biases the sensor. In the presence of an external force the cilia bend, resulting in a change of the stray field; hence, changing the sensor's resistance. (a)

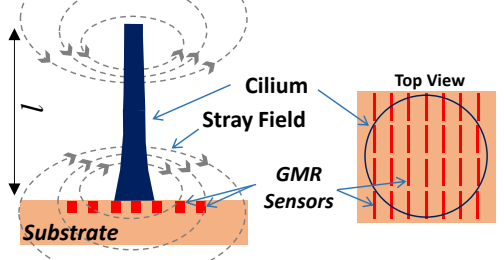

(b)

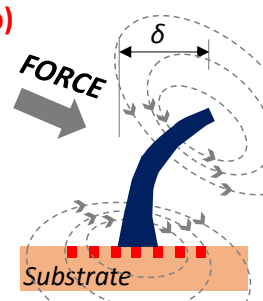

Fig. 1: Illustration of the working principle of the cilia tactile sensor. (a) The nanocomposite cilia have a stray field that affects the giant magnetoresistance (GMR) sensor and biases it to an initial resistance value. A top view of the tactile sensor design is shown (Not to scale). (b) When the cilia are deflected by applying a force, the stray field measured with the GMR sensor changes, and hence changing the resistance.

The bending motion of the artificial cilia can be described by the Euler-Bernoulli beam theory (classical beam theory), in which bending is quantified while neglecting the shear deformation of the beam $[9,21]$. Using the displacement-force relationship of an elastic cylindrical beam, the value of beam displacement $\delta$ when applying a force $F$ that has $\mathrm{x}, \mathrm{y}$ and $\mathrm{z}$ components at the free end of a beam with length $l$, Young's modulus $E$, and diameter $D$, is expressed as:

$$
\delta=\vec{F} \frac{64 l^{3}}{3 \pi E D^{4}}=\left(\vec{F}_{x}+\vec{F}_{y}\right) \frac{64 l^{3}}{3 \pi E D^{4}} .
$$

The tactile sensor consists of 4 elements (R1 to R4) separated by $0.8 \mathrm{~mm}$ and operate simultaneously. Each tactile element is made of $3 \times 3$ cilia realized on top of a GMR sensor array with $37 \times 16$ elements connected in series forming an active area of $1.25 \mathrm{~mm} \times 1.25 \mathrm{~mm}$. Each GMR sensor is 50 $\mu \mathrm{m}$ long and $3 \mu \mathrm{m}$ wide with sensitivity along the width direction.

\section{B. Fabrication}

Iron NWs with $6 \mu \mathrm{m}$ in length and $35 \mathrm{~nm}$ in diameter are fabricated by electrodeposition into a nanoporous aluminum oxide membrane, prepared by a two-step anodization process using oxalic acid on an aluminum substrate [22]. The magnetic nanocomposite is prepared by mixing NWs dispersed in Sodium dodecyl sulfate (SDS) surfactant with PDMS (Sylgard 184 Silicone Elastomer, Dow Corning Corporation) that is used as the polymeric matrix. The nanocomposite is then cured at $90^{\circ} \mathrm{C}$ for 1 hour (Fig. 2a). With this process the NWs concentration can be adjusted easily, and a volume ratio of $14 \% \mathrm{NWs} / \mathrm{PDMS}$ is chosen to avoid adversely affecting the polymerization process of the PDMS or the elasticity of the cilia. The morphology and dimensions of dispersed Fe NWs are investigated using scanning electron (Fig. 2b). A 3D reconstruction of multiple transmission electron microscopy images across $10 \mu \mathrm{m}$ thickness of a nanocomposite cilia is shown in Fig. 2c confirming the alignment of the NWs. X-ray diffraction analysis reveals the NWs are polycrystalline Fe with a thin shell of magnetite (Fig. $2 d)$.

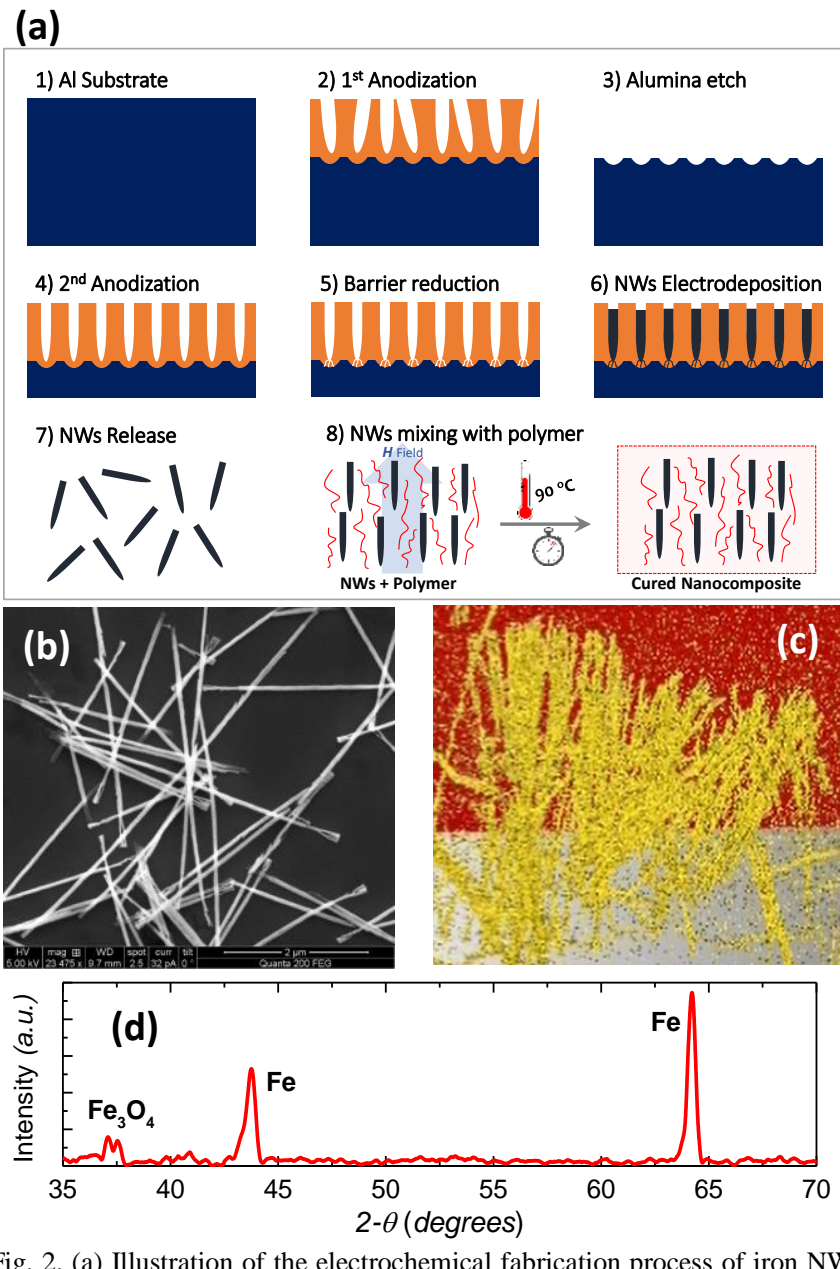

Fig. 2. (a) Illustration of the electrochemical fabrication process of iron NWs and the process of fabricating the nanocomposite. Aluminum substrate (1) is anodized (two steps) using oxalic acid to obtain uniform nanopores of $\sim 35$ $\mathrm{nm}$ in diameter, followed by a barrier reduction step for establishing an electrical contact with the bottom electrode (2-5). Iron is electrodeposited into the nanopores (6) and then the NWs are released by etching the Alumina membrane and dispersed in ethanol (7). NWs are mixed with the polymer and the nanocomposite is cured. The NWs are aligned in a certain direction by applying a homogenous magnetic field during the curing process (8). (b) Scanning electron microscopy image of dispersed Fe NWs. (c) 3D reconstruction of multiple transmission electron microscopy images across 10 $\mu \mathrm{m}$ thickness of a nanocomposite cilia with aligned NWs. The yellow lines represent the NWs inside of the polymer and the red plane is a representative cross-section plane. (d) X-ray diffraction results for a nanocomposite with $\mathrm{Fe}$ nanowires.

Four GMR sensor arrays are fabricated using standard lithography, ion beam deposition and ion milling on a silicon substrate with the following structure (thickness in $\mathrm{nm}$, compositions in atomic \%): $\mathrm{Si} / \mathrm{Al}_{2} \mathrm{O}_{3} 60 / \mathrm{Ta} 1.0 / \mathrm{Ni}_{80} \mathrm{Fe}_{20} 2.8 /$ 
$\mathrm{Co}_{90} \mathrm{Fe}_{10}$ 2.5/ Cu 2.1/ $\mathrm{Co}_{90} \mathrm{Fe}_{10}$ 2.3/ $\mathrm{Mn}_{76} \mathrm{Ir}_{24}$ 7.0/ Ta 10.0 [18]. The metallic contacts are defined by lithography and liftoff of $300 \mathrm{~nm}$ thick $\mathrm{Al}_{98.5} \mathrm{Si}_{1.0} \mathrm{Cu}_{0.5}$ and $15 \mathrm{~nm}$ thick $\mathrm{Ti}_{10} \mathrm{~W}_{90}\left(\mathrm{~N}_{2}\right)$ layers.

A thin PDMS layer of $\sim 5 \mu \mathrm{m}$ is then spun on top of the substrate to provide electrical isolation and, at the same time, enhance the adhesion of the cilia to the GMR sensor.

A master mold technique is used to pattern the nanocomposite cilia. The master mold is a $1 \mathrm{~mm}$ thick poly(methylmethcrylate) (PMMA), into which an array of holes with $200 \mu \mathrm{m}$ in diameter is patterned with a $\mathrm{CO}_{2}$ laser cutter (Universal PLS6.75). The nanocomposite is casted onto the surface of the GMR sensor, and the master mold is mounted on top, thereby aligning the holes with the GMR sensors using a microscope. This structure is then placed in a desiccator for 30 minutes to remove any trapped air bubbles and assist in filling the pores. Next, the composite is cured at 90 degrees Celsius for one hour with an external magnetic field along the horizontal direction of the cilia, forming the cilia with aligned NWs on top of the GMR sensor. After releasing the cured cilia, they are fully magnetized by applying a magnetic field of $10 \mathrm{kOe}$. The permanent magnetic properties of the nanocomposite have been shown to be stable and corrosion resistant [9]. The fabrication process is illustrated in Fig. 3.

(a)

Photoresist exposure and development Photoresist Substrate
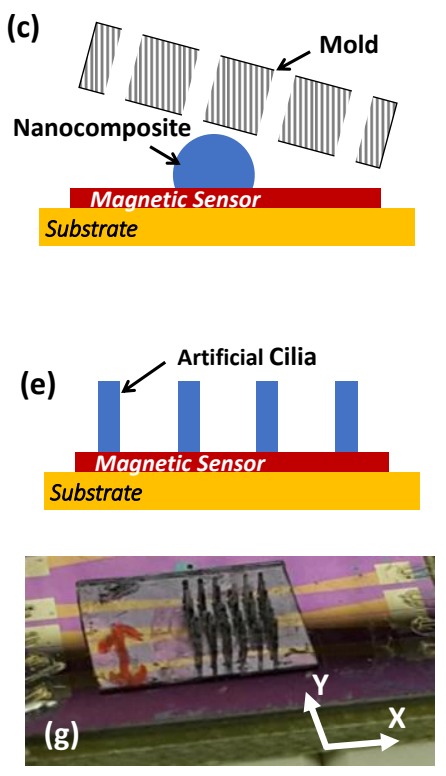

(d)
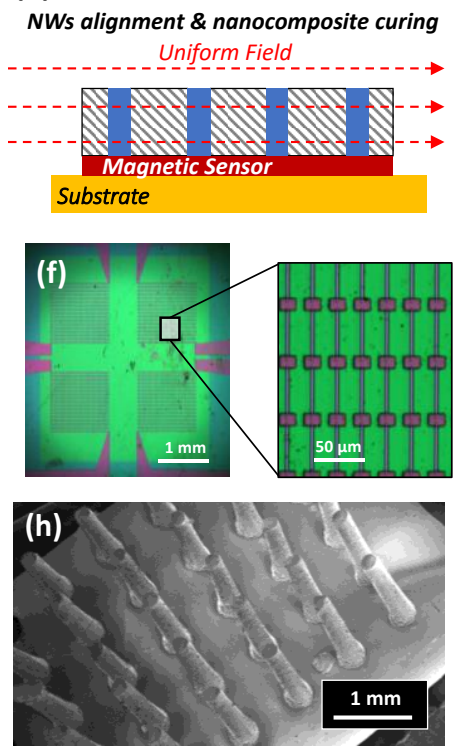

Fig. 3. Fabrication process of the magnetic cilia tactile sensor. (a,b) GMR sensors are fabricated using standard lithography process, ion beam deposition and ion milling on a silicon substrate. (b-e) Cilia are fabricated using a PMMA mold technique, and applying a homogeneous magnetic field aligns the NWs. (f) Microscope image of the fabricated GMR sensor array prior to cilia integration. (g) Optical image of the fabricated sensors. (h) Scanning Electron Microscopy image of a cilia array. Each cilium is $1 \mathrm{~mm}$ in length and $200 \mu \mathrm{m}$ in diameter.

\section{Characterization}

In order to study the magnetic properties of the nanocomposite cilia and confirm the permanent magnetic behavior, the magnetization curves along the cilia and in the perpendicular direction are obtained using a vibrating sample magnetometer (MicroMag 3900).

Since obtaining high elasticity of the nanocomposite cilia is crucial to increase the sensitivity of the sensor, a study is carried out to investigate the effect of the NW concentration on the elasticity. The Young's modulus of the nanocomposite cilia is obtained by fixing them between the holder plate and the moveable head of an extensometer tensile/compressive force testing system (INSTRON 5966) as shown in Fig. 4. The system can precisely determine the exerted force and the vertical displacement. By using a $10 \mathrm{~N}$ load cell and three cilia that are $1 \mathrm{~mm}$ long and $200 \mu \mathrm{m}$ in diameter, resolutions of 5 $\mathrm{mN}$ and $16 \mu \mathrm{m}$ are obtained for the tensile force and vertical displacement, respectively. The extensometer is calibrated after mounting the cilia and then tensile forces are applied to the cilia until achieving a strain of 1.3 (130\% stretching). The Young's modulus is calculated as the ratio between stress and strain (slope) in the elastic (linear) region.
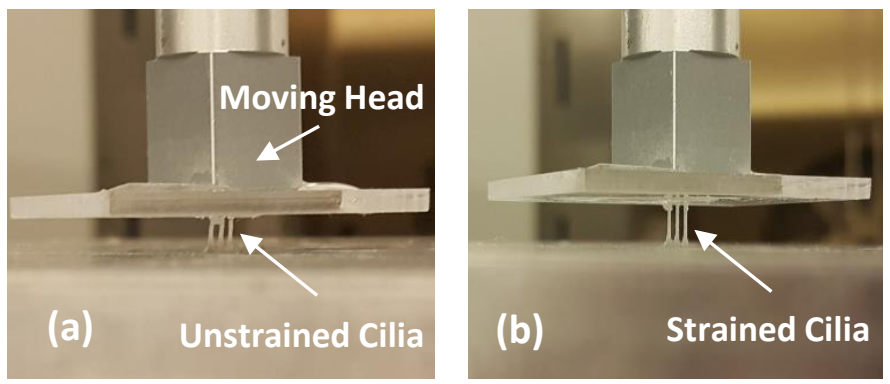

Fig. 4. Stress-strain experiment to study nanocomposite elasticity showing (a) cilia in their initial state and (b) at $100 \%$ stretch (strain=1).

In order to characterize the GMR sensor array, a Helmholtz coil is used to apply a magnetic field up to $18 \mathrm{Oe}$, while the resistance is measured with a source meter (Keithley 2400) at a DC current of $100 \mu \mathrm{A}$. The fields are applied in the sensitive and non-sensitive directions of the GMR sensors.

The performance of the four tactile sensors arrays with 1 $\mathrm{mm}$ long and $200 \mu \mathrm{m}$ in diameter cilia is studied by applying vertical forces while measuring the sensor response. The forces are applied using the computer controlled force testing system mentioned earlier, which can precisely determine the amount of force applied to the sensors. The resistance variation upon the deflection of the cilia is studied by powering the sensors with $100 \mu \mathrm{A}$ DC current $\left(I_{D C}\right)$ using current source (Keithley 2400), and measuring the voltage across each of the sensors in the array $\left(V_{R 1}, V_{R 2}, V_{R 3}, V_{R 4}\right)$ using a data acquisition system (NI USB-6229) controlled using a LabVIEW program. The experimental setup is illustrated in Fig. 5 where the four tactile sensors are referred to as R1, R2. R3 and R4. 


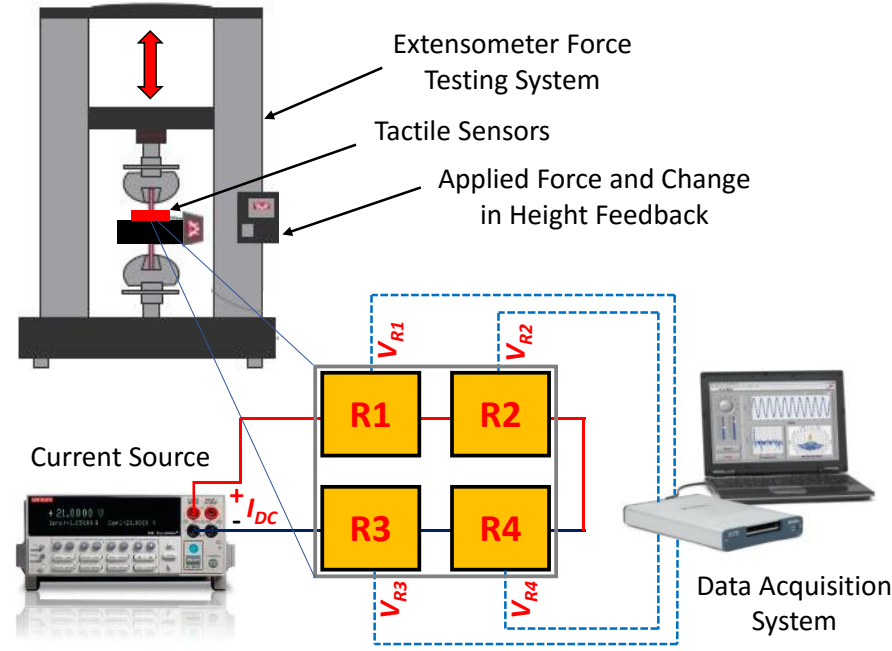

Fig. 5. Experimental setup for the four-element tactile sensor array.

\section{RESULTS}

Magnetic characterization for the nanocomposite cilia is conducted by obtaining the magnetizations curves as shown in Fig. 6. The results show that the cilia with horizontally aligned NWs have a magnetic anisotropy with a remanence to saturation magnetization $(\mathrm{M} / \mathrm{Ms})$ of $70 \%$ and $19 \%$ along the perpendicular and parallel direction, respectively, with a coercivity of $1.5 \mathrm{kOe}$. These results show the permanent magnetic-like behavior of the nanocomposite and confirm the alignment of the NWs.

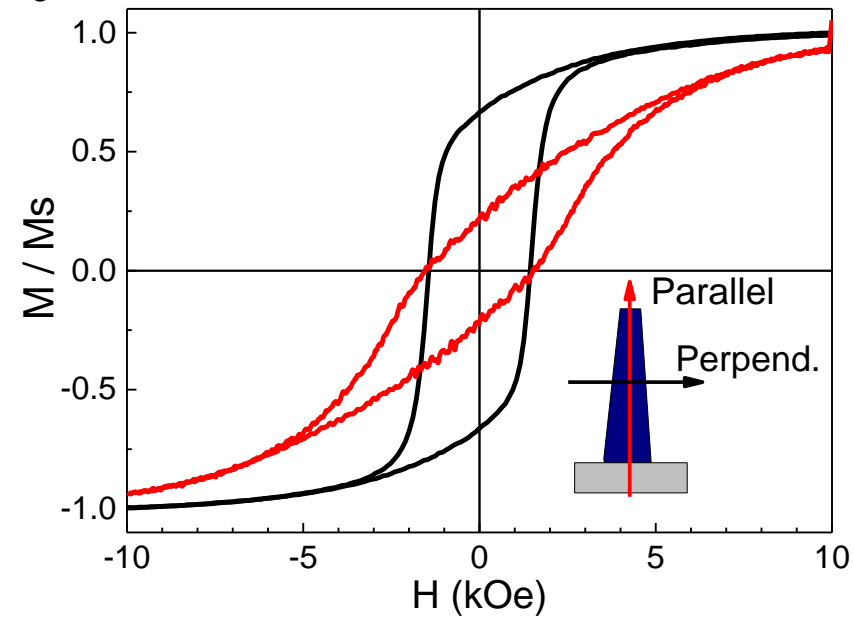

Fig. 6. Nanocomposite magnetization curves obtained with a vibrating sample magnetometer in the vertical (parallel) and horizontal (perpendicular) direction of a cilia array. The NWs in the cilia are aligned horizontally.

The nanocomposite cilia have a Young's modulus of 0.76 $\mathrm{MPa}$, compared to the pure PDMS cilia that have a Young's modulus of $0.71 \mathrm{MPa}$. Thus, incorporating NWs in the nanocomposite does not have a significant effect on the rigidity of the cilia, and the advantage of the high elasticity of the PDMS is maintained.

The GMR sensor array characterization in Fig. 7 shows a linear response to homogeneous magnetic field between 0-23 Oe with sensitivities of $57 \Omega / \mathrm{Oe}$ and $4.8 \Omega / \mathrm{Oe}$ in the sensitive and non-sensitive directions, respectively. This anisotropic behavior equips the cilia sensor array with a directional sensitivity.

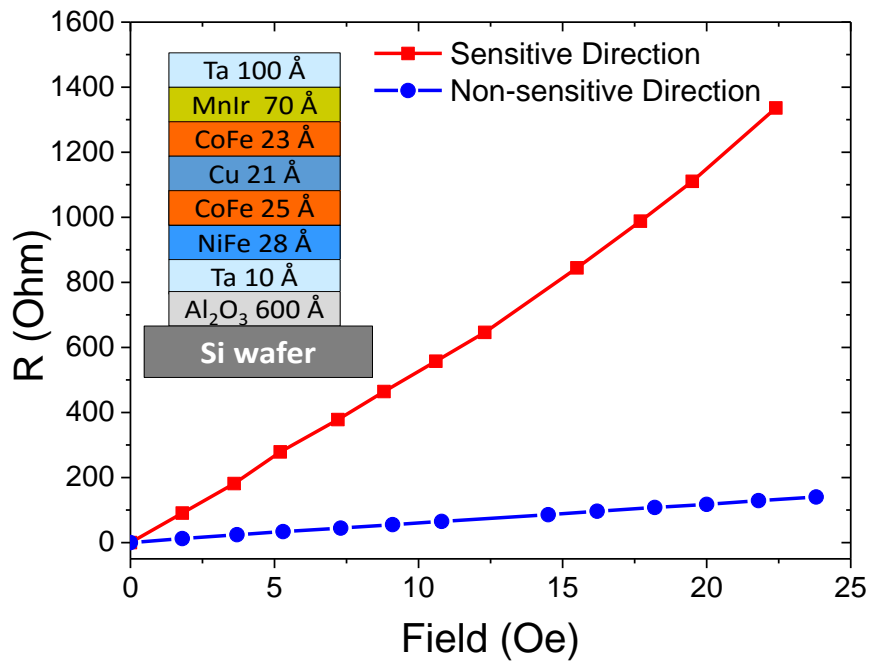

Fig. 7. Resistance response of the GMR sensor array with an external magnetic field applied to the sensitive and non-sensitive directions. Inset: fabricated GMR sensor stack.

The cilia sensor response to external forces is shown in Fig. 8 . The results show the sensor's ability to detect vertical forces up to $55 \mathrm{mN}$ with a sensitivity of $0.8 \mathrm{mV} / \mathrm{mN}$. The resolution is obtained by considering the noise in the test setup and is found to be $6 \mathrm{mN}$. A reference measurement for cilia without NWs revealed that there is almost no effect of pressure on the GMR sensor.

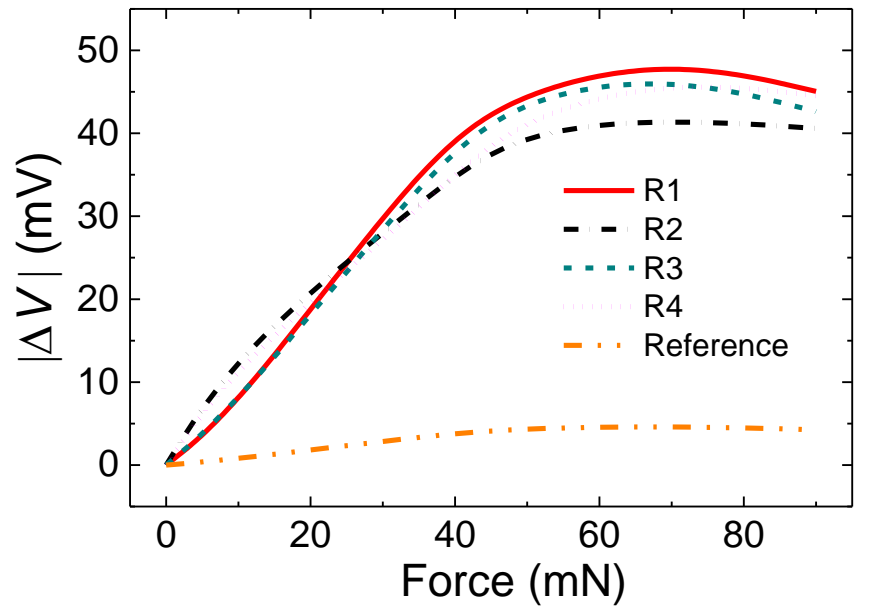

Fig. 8. Tactile sensor response for different forces.

The developed sensor was put to the test by using it for reading Braille letters. Each Braille letter consists of 6 palpable dots arranged in two rows and 3 lines [23]. Fig. 9 shows Braille letters forming the word "KAUST" and the signal from the tactile sensor when brought in contact with the letters. Two sensors (R1 \& R2) are used for the simultaneous detection of the dots in one row, and the entire letter is read in 3 steps, i.e. the 3 rows are read sequentially. The other two sensors (R3 \& R4) are used to confirm the signal pattern and remove background noise. The maximum obtained voltage change when detecting the dots is $35 \mathrm{mV} \pm 5 \mathrm{mV}$ which corresponds to around 6 Oe field change upon cilia deflection according to the GMR sensor characterization results in Fig. 7. The response in Fig. 9 shows clear pattern allowing easy 
determination of the Braille characters and the corresponding letter.
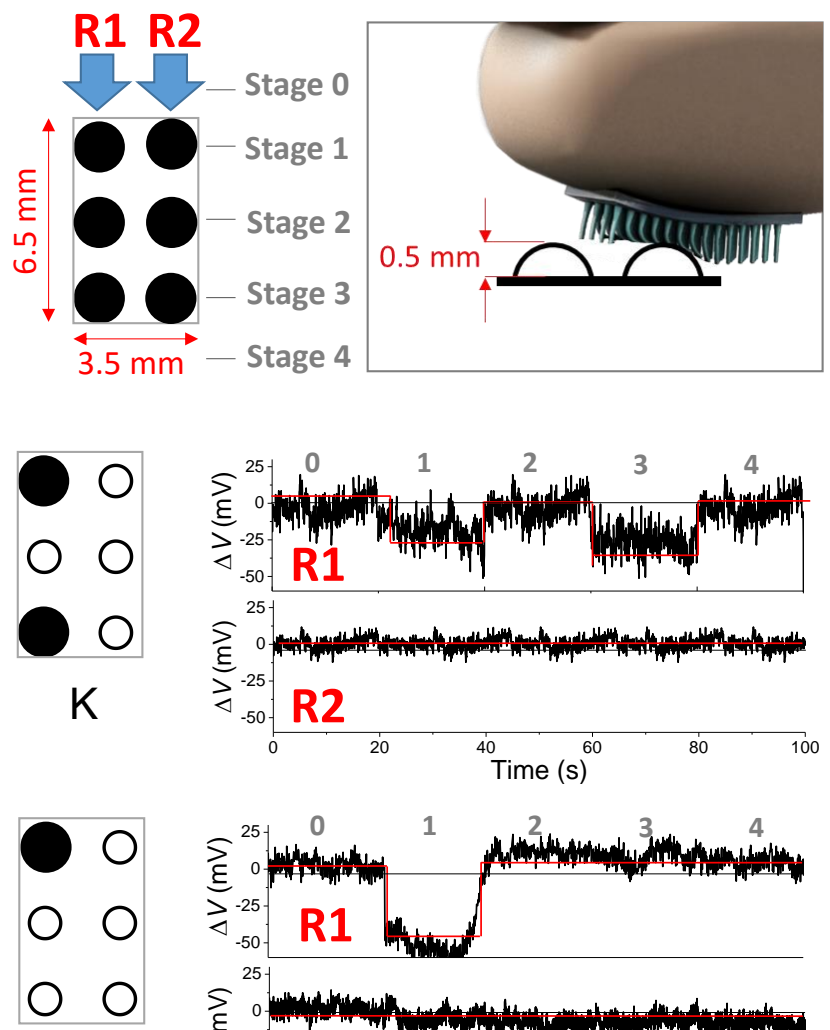

A
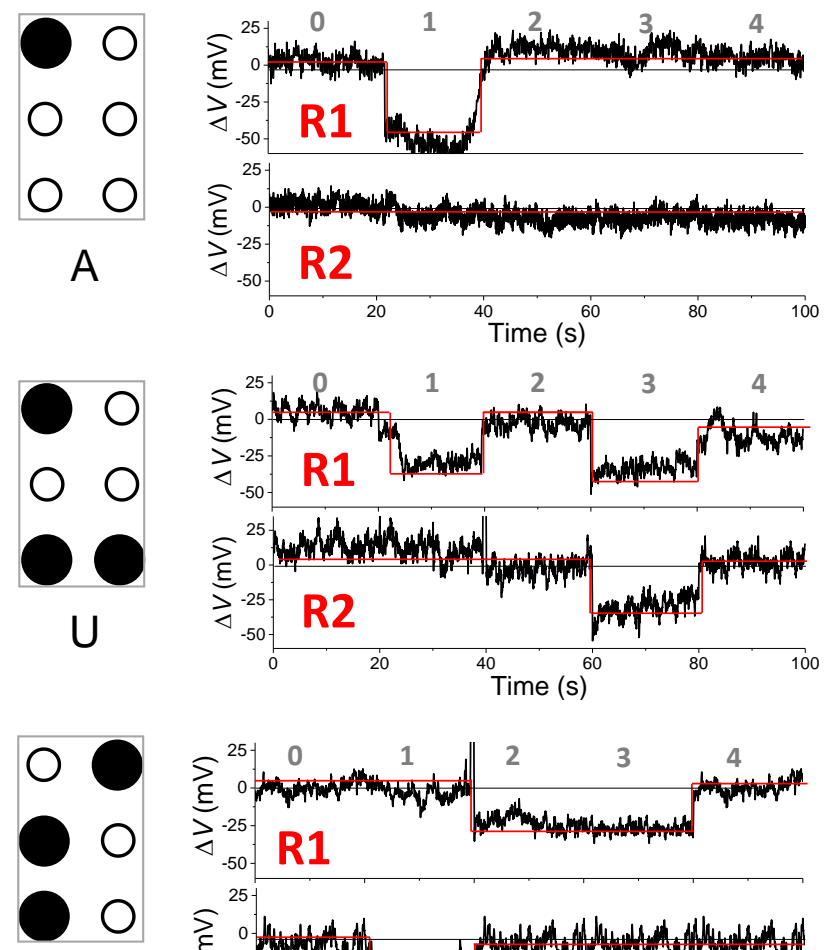

$S$
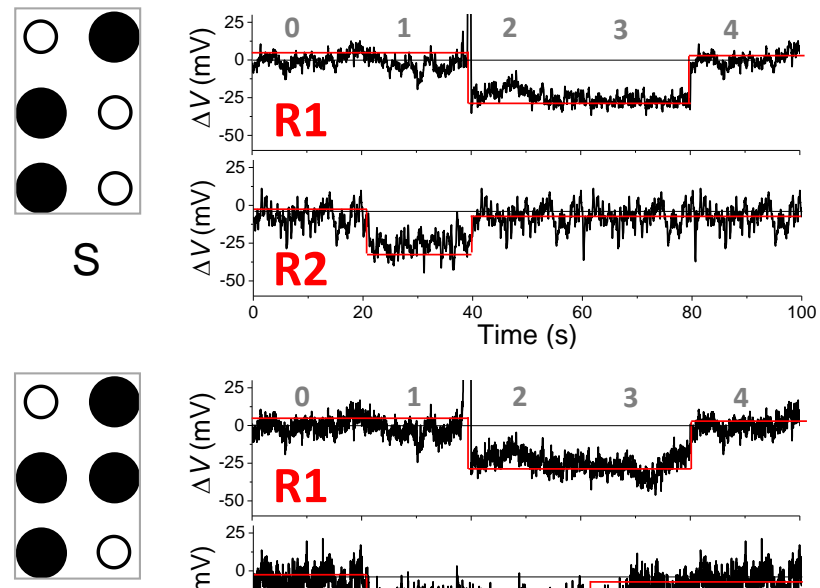

$\mathrm{T}$

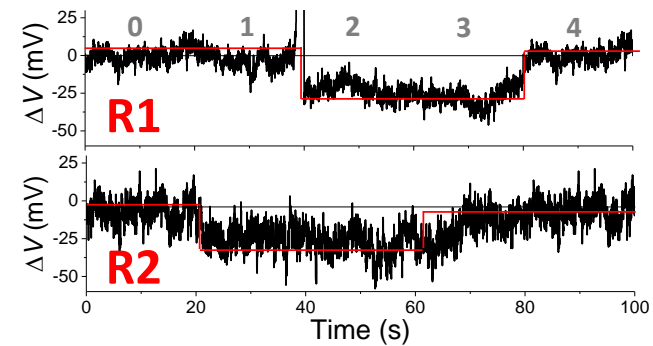

Fig. 9. Braille characters and the signals obtained from the sensor while reading the letters of the word "KAUST".

\section{CONCLUSION}

A novel NWs-based permanent magnetic and highly elastic nanocomposite cilia tactile sensor has been realized and has been used for Braille characters reading. GMR sensors detect the change of the stray field created by the permanent magnetic cilia when deflected, in particular by contacting the dots of Braille characters. Each dot can be recognized with a high sensitivity and the utilized scheme of 4 tactile sensing elements enables reading a Braille letter in 3 steps. This promising result could possibly be further improved by increasing the number of sensor elements to optimize the sensor design with respect to the reading speed.

A distinct advantage of the cilia is the permanent magnetic behavior of the iron NWs, eliminating the need for an external magnetic field to magnetize the cilia, enabling a compact and power-efficient device design. The tactile sensors' characteristics can be tailored in a wide range by simply modifying the dimensions of the cilia, the choice of the materials as well as by the concentration of the NWs. Previously, GMR sensors have be fabricated on ultra-thin flexible substrates [17], which can be utilized to realize conformal and bendable cilia sensors in the future.

\section{ACKNOWLEDGEMENTS}

Research reported in this publication is supported by the King Abdullah University of Science and Technology (KAUST).

\section{REFERENCES}

[1] Y.-L. Park, B. Chen, and R. J. Wood, "Design and fabrication of soft artificial skin using embedded microchannels and liquid conductors," IEEE Sens. J., vol. 12, no. 8, pp. 2711-2718, 2012.

[2] S. C. B. Mannsfeld, et al., "Highly sensitive flexible pressure sensors with microstructured rubber dielectric layers," Nature Mater., vol. 9, pp. 859, 2010.

[3] G. Schwartz, B. C. -K. Tee, J. Mei, A. L. Appleton, D. H. Kim, H. Wang, Z. Bao, "Flexible polymer transistors with high pressure sensitivity for application in electronic skin and health monitoring," Nature Comm., vol. 4, pp. 1859, 2013.

[4] K. Takei, et al., "Nanowire active-matrix circuitry for low-voltage macroscale artificial skin," Nature Mat., vol. 9, pp. 821, 2010.

[5] S. Mohsin, et al., "A review of tactile sensing technologies in biomedical engineering," Sens. Actuators A, vol. 179, pp. 17, 2012.

[6] C. Pang, et al., "A flexible and highly sensitive strain-gauge sensor using reversible interlocking of nanofibres," Nature Mat., vol. 11, pp. 795, 2012.

[7] M. Hein , et al., "Fabrication of bio inspired inorganic nanocilia sensors," IEEE Trans. on Magnetics, vol. 49, pp. 191, 2013.

[8] C. Liu, "Micromachined biomimetic artificial haircell sensors," Bioinspir. and Biomim., vol. 2, pp. S162, 2007.

[9] A. Alfadhel, et al., "Magnetic nanocomposite for biomimetic flow sensing," Lab Chip, vol. 14, pp. 4362, 2014.

[10] M. Y. Alnassar, Y. P. Ivanov, and J. Kosel, "Fabrication and Properties of Multiferroic Nanocomposite Films," IEEE Trans. Mag., vol. 51, pp. 2500504, 2015.

[11] M. Y. Alnassar, A. Alfadhel, Y. P. Ivanov, and J. Kosel, "Magnetoelectric Polymer Nanocomposite for Flexible Electronics," Journal of Applied Physics, vol. 117, pp. 17D711, 2015. 
[12] M. Suter, et al., "A photopatternable superparamagnetic nanocomposite: Material characterization and fabrication of microstructures," Sens. and Actuat. B, vol. 156, pp. 433, 2011.

[13] B. Zhou, et al.," Design and fabrication of magnetically functionalized flexible micropillar arrays for rapid and reversible microfluidic mixing," Lab Chip, vol. 15, pp. 2125-2132, 2015.

[14] A. Alfadhel, and J. Kosel, "Magnetic Nanocomposite Cilia Tactile Sensor," Adv. Mater., vol. 27, pp. 7888-7892, 2015.

[15] A. Alfadhel, and J. Kosel, "Magnetic Micropillar Sensors for Force Sensing," 2015 IEEE Sensors Applications Symposium (SAS), Zadar, Croatia, 12-15 April, 2015.

[16] S. P. Parkin, "Giant magnetoresistance in magnetic nanostructures," Annu. Rev. Mater. Sci., vol. 25, pp. 357-388, 1995.

[17] M. Melzer, et al., "Imperceptible magnetoelectronics," Nature Comm., vol. 6, pp. 6080, 2015.

[18] P. Freitas, R. Ferreira, S. Cardoso, and F. Cardoso, "Magnetoresistive sensors", J. of Physics: Condens. Matter, vol. 19, pp. 165221, 2007.

[19] D. Karnaushenko, D. Makarov, C. Yan, R. Streubel, and O. Schmidt, "Printable Giant Magnetoresistive Devices," Adv. Mater., vol. 24, pp. 4518-4522, 2012.

[20] M. Pannetier, C. Fermon, G. Goff, J. Simola J, E. Kerr, "Femtotesla magnetic field measurement with magnetoresistive sensors," Science, vol. 304, Issue 5677, pp. 1648-1650, 2004.

[21] S. Timoshenko, "History of strength of materials," McGraw-Hill New York, 1953.

[22] K. Nielsch, F. Müller, A. P. Li and U. Gösele, "Uniform Nickel Deposition into Ordered Alumina Pores by Pulsed Electrodeposition," Adv. Mater., vol. 12, pp. 582, 2000.

[23] L. Braille, "Method of Writing Words, Music, and Plain Songs by Means of Dots, for Use by the Blind and Arranged for Them," Paris, France: National des Jeunes Aveugles [National Institute for Blind Children], 1829. 